\title{
Master-Slave Node Method of Processing Plane Node
}

\author{
SU Zhi-Gang ${ }^{1, a}$, WANG Fei ${ }^{1, b}$, LI Qing-Hua ${ }^{1, \mathrm{c}}$, SHANG Wei-Fang ${ }^{2, \mathrm{~d}}$, \\ ZHANG Zi-Fu ${ }^{1, e}$ \\ ${ }^{1}$ China Electric Power Research Institute, Xuanwu District, Beijing 100055, China \\ ${ }^{2}$ China railway 22 second engineering bureau group co., LTD, 100043

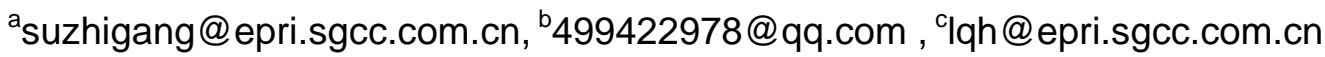 \\ d616197637@qq.com, ${ }^{\mathrm{d} z z f @ e p r i . s g c c . c o m . c n}$
}

Keywords: Plane node; Truss structure; Method of master-slave node; Stiffness matrix

Abstract: In the process of finite element analysis about space truss structure, the problem of plane node must be dealt with. Generally, there are four methods about plane node, void- rod element method, removal freedom method, adding virtual spring method, method of beam element. In this paper a new treatment method of master-slave node is developed. The plane node unstable direction degrees of freedom and the direction of the master node degrees of freedom are tied together. This method has strong stability, will not affect the distribution of force in the model with high stability calculation.

\section{Foreword}

In the process of finite element analysis about space truss structure, the problem of plane node must be dealt with. All rod elements in the space truss which are crossing the same node are placed on the same plane, and there is no any rod element at direction of this node vertical to the plane, such node is called as the plane node ${ }^{[1]}$. This ideal space truss system is a geometrical variable system, stiffness matrix of the structure will become a strange matrix, and such space truss system isn't solved, therefore such situate shall be treated through analysis.

Generally, there are four methods about the zero stiffness plane node, void- rod element method, removal freedom method, adding virtual spring method, method of beam element ${ }^{[2]}$.

The void-rod element method is to add the void rod element between the plane node and the near stable node. This is a good traditional method during linear analysis. But if stiffness of the void rod isn't high enough, this method will cause a unstable model during non-linear analysis. The void rod member proper body needs a very small stiffness (such as a very small cross section), it will not affect distribution of force in the model.

The removal freedom method is applicable to the following two conditions: one is exiting plane direction consistent with any three dimension direction, another is that analysis is linear. Under this condition, instability problem is solved through removing freedom of the node at instable direction. The node force which is obtained via the method making the node stable is correct during linear analysis, but position of the node which freedom is removed in this method is obviously incorrect.

Adding virtual spring method is to add the virtual spring with small axial stiffness at $\mathrm{x}, \mathrm{y}$ and $\mathrm{z}$ directions of the all nodes, this method will avoid appearance of zero stiffness at the plane node location. The instable node or the mechanism which displacements are unreasonable can be found by this method. Stability of the instable node and the mechanism can be improved. But if this method is applied in the non-linear analysis, this method may cause the instable model.

In the beam and rod element mixing unit method, the beam unit is applied to replace some rod element unit with the plane node. The beam unit can provide certain stiffness at $\mathrm{x}, \mathrm{y}$ and $\mathrm{z}$ three reverse directions, which makes the plane node stable. Analysis speed may be reduced if the beam unit analysis calculation is applied, but it is very beneficial to eliminate the plane node and the mechanism. 


\section{Key technology}

In order to solve strange matrix problem of the plane node existing in the finite element model of the space truss structure, the master-salve node method is applied in combining with the void-rod member method and the removal freedom method ${ }^{[3][4]}$, the freedom direction of the plane node with stiffness of zero is bonded with the same freedom direction of the stable node which is mostly near to this plane node.

\subsection{Working principle}

General node stiffness matrix equation ${ }^{[5]}$ which shall be solved during finite element analysis of the rod member system is:

$$
[K]\{\Delta\}=\{P\}
$$

$[K]$ Stiffness matrix of general node

$\{\Delta\}-$ Displacement vector of general node

$\{P\}$ L Load vector of comprehensive node

In order to solve stiffness matrix equation of the general node, arrange the displacement vector element of the general node again according to the boundary restraint conditions, the line and the row of the stiffness matrix of the general node $[K]$ corresponding to displacement shall be arranged again.

Re-arrangement form of the displacement vector of the general node becomes:

$$
\left\{\frac{\left\{\Delta_{D}\right\}}{\left\{\Delta_{R}\right\}}\right\}
$$

Re-arrangement form of the stiffness matrix of the general node becomes:

$\left[\begin{array}{ll}K_{D D} & K_{D R} \\ K_{R D} & K_{R R}\end{array}\right]$

In which, the subscript $\mathrm{D}$ means non-restraint displacement, and the subscript $\mathrm{R}$ means restraint displacement.

$$
\left[\begin{array}{ll}
K_{D D} & K_{D R} \\
K_{R D} & K_{R R}
\end{array}\right]\left\{\frac{\left\{\Delta_{D}\right\}}{\left\{\Delta_{R}\right\}}\right\}=\left\{\frac{\left\{P_{D}\right\}}{\left\{P_{R}\right\}}\right\}
$$

After the restraint conditions are introduced, $\left\{\Delta_{R}\right\}=0$, stiffness matrix equation of the general node is spread as:

$$
\left[K_{D D}\right]\left\{\Delta_{D}\right\}=\left\{P_{D}\right\}
$$

If $K_{D D}$ is non strange matrix, it will be a reversible matrix. Non restraint displacement is obtained from this formula, and the stiffness matrix equation of the general node is solved.

All rod elements in the space truss which are crossing the same node are placed on the same plane, and there is no any rod element at direction of this node vertical to the plane, such node is called as the plane node. Shown as No 5 node in figure 1. Such ideal space truss structure is a reversible system. After the stiffness matrix element of the general node $[K]$ is arranged again according to the boundary restraint conditions. $K_{D D}$ is a strange matrix before insufficient boundary restraint conditions. Now the stiffness matrix equation of the general node can’t be solved, condition of such plane node shall be treated. 


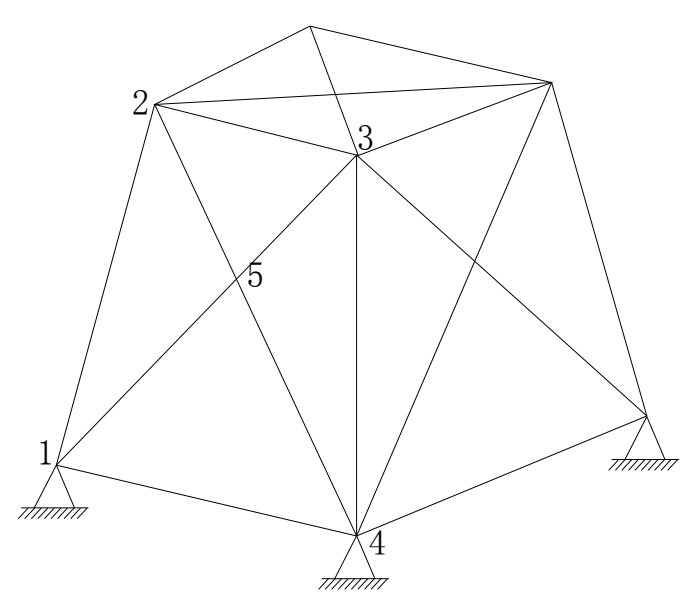

Fig. 1 Plane node of space truss

\subsection{Flow chart of method}

Flow chart of the treatment method for the plane node is shown as figure 2. First step, search the plane node of the finite element model of the space truss structure rod element unit. Second step, determine instable direction of the above mention plane node. Third step, determine the master node attached on the plane node. Fourth step, bind the instable direction of the plane node with the master node.

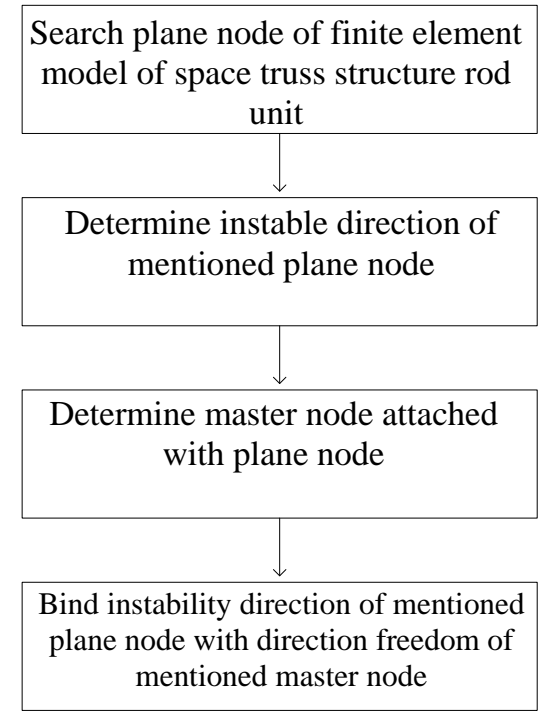

Fig. 2 Flow chart for treatment method of plane node

\subsection{Technical realization}

(1) Search plane node

Set $\mathrm{a}, \mathrm{b}$ and $\mathrm{c}$ are three vectors in the space, and then $(\mathrm{a} \times \mathrm{b}) \cdot \mathrm{c}$ is called as mixing product of three vectors $a, b$ and $c$, which is written $a s(a, b, c)$. Geometrical meaning of $|(a \times b) \cdot c|$ means volume of the parallel hexahedron which takes $a, b$ and $c$ as edges. In case of $(a, b, c)=(a \times b) \cdot c=0$, it means normal vector of the plane which constitutes of $a$ and $b$ is vertical to $c$, it means $c$ is also located on the plane which constitutes of $\mathrm{a}$ and $\mathrm{b}$. Of course, $\mathrm{c}$ may be 0 , and 0 shares the plane with any vector. Therefore in case of $(a, b, c)=0$, then three vectors of $a, b$ and $c$ share the plane. In case of vector $a=(\cos x 1$, cosy1, $\operatorname{cosz} 1)$, vector $b=(\cos x 2, \cos y 2, \operatorname{cosz} 2)$ and vector $c=(\cos x 3, \cos y 3, \cos z 3)$, 


$$
\begin{aligned}
& (a, b, c)=(a \times b) \cdot c=\left[\begin{array}{lll}
\cos x_{1} & \cos y_{1} & \cos z_{1} \\
\cos x_{2} & \cos y_{2} & \cos z_{2} \\
\cos x_{3} & \cos y_{3} & \cos z_{3}
\end{array}\right] \\
& =\cos x_{3} \cdot\left(\cos y_{1} \cdot \cos z_{2}-\cos z_{1} \cdot \cos y_{2}\right) \\
& +\cos y_{3} \cdot\left(\cos z_{1} \cdot \cos x_{2}-\cos x_{1} \cdot \cos z_{2}\right) \\
& +\cos z_{3} \cdot\left(\cos x_{1} \cdot \cos y_{2}-\cos y_{1} \cdot \cos x_{2}\right)=0
\end{aligned}
$$

The vectors a, b and c share the plane.

When one point is judged whether it is the plane node or not, firstly find out all rod elements corresponding to this node, and then judge whether all rod element are located on the same plane. Judge whether all rod elements are located on the same plane. Only two pieces are freely selected, use the rod element 1 and the rod element 2 to determine one plane, and judge whether other rod elements are located on this plane in turn. If one rod element isn't located on this plane, this node isn't the plane node.

Find out cosine values of the angle between the rod element 1 and the coordinate axis which are $\cos x 1$, cosy 1 and cosz 1 respectively, vector a of the rod element $1=(\cos x 1$, cosy $1, \cos z 1)$. Cosine values of the angle between the rod element 2 and the coordinate axis which are cosx2, cosy2 and $\operatorname{cosz} 2$ respectively, vector $b$ of the rod element $=(\cos x 2$, cosy2, $\cos z 2)$. Cosine values of the angle between the rod element 3 and the coordinate axis which are cosx3, cosy3 and cosz 3 respectively, vector c of the rod member $3=(\cos x 3$, cosy3, cosz3)

$$
\begin{aligned}
& c_{1}=\cos y_{1} \cdot \cos z_{2}-\cos z_{1} \cdot \cos y_{2} \\
& c_{2}=\cos z_{1} \cdot \cos x_{2}-\cos x_{1} \cdot \cos z_{2} \\
& c_{3}=\cos x_{1} \cdot \cos y_{2}-\cos y_{1} \cdot \cos x_{2}
\end{aligned}
$$

If $\cos x_{3} \cdot c_{1}+\cos y_{3} \cdot c_{2}+\cos z_{3} \cdot c_{3}=0$, the rod element 3 is located on the plane which constitutes of the rod element 1 and the rod element 2. If all rod elements are located on the same plane, this node is a plane node. After the node is judged as the plane node, save the plane node in the plane node database.

(2) Determine instable direction of plane node

If the node is a plane node, find out maximum value of absolute value of $c_{1}, c_{2}$ and $c_{3}$. If absolute value of $c_{1}$ is maximum value, $x$ direction is instable direction. If absolute value of $c 2$ is maximum value, y direction is instable direction. If absolute value of c3 is maximum value, z direction is instable direction.

(3) Find out master node

Find out the node mostly near to the plane node, judge whether this node is stored in the plane node database. If this node is stored in the plane node database, further search the node mostly near to the plane node. If this node isn't stored in the plane node database, the non-plane node which is mostly near to the plane node is taken as the master node, the plane node is taken as the slave node.

(4) Restraint freedom

The freedom at instable direction of the plane node is bonded with the freedom at this direction of the master node. Now boundary constraint conditions are sufficient, it is a non-strange matrix. Stiffness matrix equation of the master node can be solved, condition of the plane node is solved.

\subsection{Sample}

Figure 3 is schematic figure for treatment of the plane node with the master-salve node method of the space truss structure. In which, no 1743 node is a plane node, no 1703 node is master node of this plane node, $\mathrm{z}$ direction of no 1743 plane node is instable direction, freedom of no 1743 node at $\mathrm{z}$ direction is bonded with freedom of no 1703 node at $\mathrm{z}$ direction by the master-slave node method. 


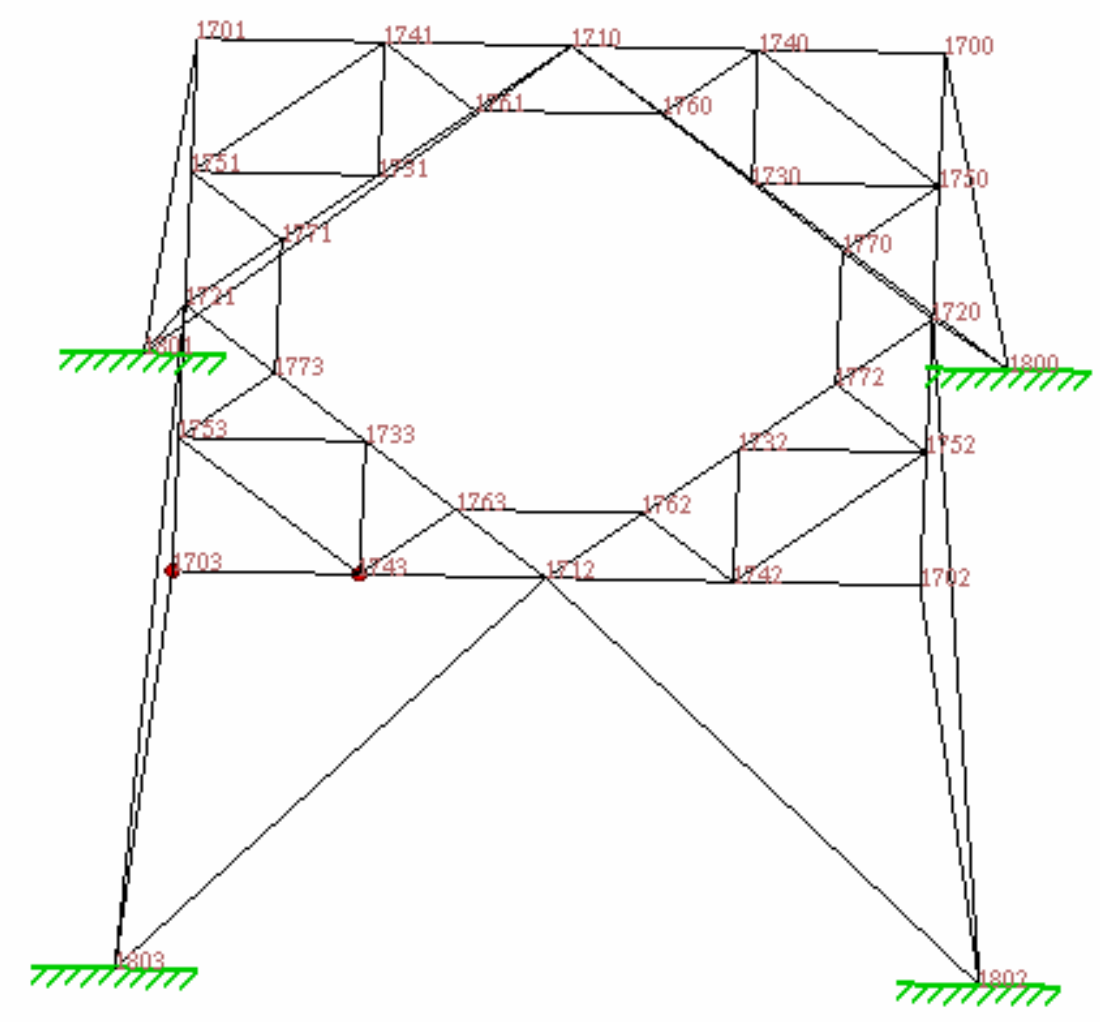

Fig. 3 Plane node treatment with master-slave node method of space truss structure

\section{Advantage of master-slave node method}

Compared with the current plane node treatment method, the master-slave node method for treatment of the plane node has the following advantages:

1. The master-slave node method for treatment of the plane node is mainly applied in finite element analysis of the space truss unit. It combines the void rod element method and the removal freedom method, which deal with appearance of the structure stiffness matrix at plane node location of the truss structure well;

2. Solve instability problem during non-linear analysis;

3. Not affect distribution of force in the model, and no great sudden change of displacement appears as the removal freedom method;

4. High calculation efficiency, which is easy to realize;

5. The plane node treatment method which has less influence on displacement has great meaning for finite element analysis of the space truss structure.

\section{Conclusion}

A new plane node treatment method of the master-salve node method is developed in this paper. It firstly search the plane node in the finite element model of the rod element unit of the space truss structure in this method, and then determine instable direction of the mentioned plane node. And then determine the master node attached on the plane node. Finally bind the instable direction of the plane node with the master node.

The void rod element method is combined with the removal freedom method in this method, which deals with appearance of the structure stiffness matrix at the plane node location of the truss structure well, which is characterized with strong stability and high calculation stability, it will not affect distribution of force in the model. 


\section{References:}

[1] Hu Guoyong. Treatment method study of plane node in power transmission tower structure [J]. Science Broadcast. 2013(10):114-116.

[2] Dong Xugang. Finite element analysis and optimization design study of truss structure [D].Xian University of Technology 2005

[3] Li Huanping, Dang Jianming. Application of master-slave node method in re-construction engineering of steel structure[J]. Science Times, 2015(5).

[4] Meng Xianda, Ge Chundi. Concept and application of master-slave node in finite element method[J]. Port engineering technology,1989,2:002.

[5] Ouyang Keqing. Large displacement stiffness matrix of space truss [J]. Journal of Tongji university,1982,4:27-38. 